\title{
Resiliencia de docentes en distanciamiento social preventivo obligatorio durante la pandemia de COVID-19

\author{
Resiliencia docente en COVID-19
}

\author{
Fabián Román ${ }^{1 \star 2 \star 4 * 5}$, Anna Forés Miravalles ${ }^{1 \star 2}$, Ismael Calandri ${ }^{1 \star 5}$, \\ Rosalba Gautreaux ${ }^{1}$, Alejandro Antúnez ${ }^{1}$, Dalul Ordhei ${ }^{\star * 6}$, Lenin \\ Calle $^{1}$, Victoria Poenitz ${ }^{1 * 7}$, Kethy Luz Correa Pérez ${ }^{1 * 9}$, Sandra \\ Torresi ${ }^{1}$, Ernesto Barceló ${ }^{1 * 4}$, Mauricio Conejo ${ }^{1 * 8}$, Ricardo Allegri ${ }^{13^{* 4 * 5}}$ \\ y Veerle Ponnet ${ }^{1}$
}

\begin{abstract}
*Correspondencia:
Fabián Román

faromanmd@gmail.com

Citación:

Román F, Fores A, Calandri

I, Gautreaux R, Antúnez A

Ordehi D, Calle L, Poenitz V,

Correa KL, Torresi S, Barcelo

E, Conejo M, Ponnet V, Allegri

R. Resiliencia en docentes en

distanciamiento social preventivo

obligatorio durante la pandemia

de COVID-19.JONED. Journal of

Neuroeducation. 2020; 1(1); 76-87.

doi: 10.1344/joned.v1i1.31727

Fuentes de financiación

Esta investigación se realizó con recursos financieros propios de los autores. Las instituciones a través de sus redes facilitaron su difusión entre los docentes.
\end{abstract}

Conflicto de interés

Los autores declaran la ausencia de conflicto de interés derivado de este trabajo.

Contribuciones de los autores Todos los autores contribuyeron de manera similar en el presente trabajo.

Editora:

Marcel Ruiz Mejías (Universitat Pompeu Fabra, España)

Revisores:

Marta Ligioiz Vázquez (Independiente, Sevilla, España) y Marcel Ruiz Mejías (Universitat Pompeu Fabra, Espanya)

El manuscrito ha sido aceptado por todos los autores, en el caso de haber más de uno, y las figuras, tablas e imágenes no están sujetos a ningún tipo de Copyright.
${ }^{1}$ Red Iberoamericana de Neurociencia Cognitiva, info@fhneurociencia.org

${ }^{2}$ Cátedra de Neuroeducación de la Universidad de Barcelona, annafores@ub.edu

${ }^{3}$ Fleni, Fundación de Lucha contra enfermedades neurológicas en la infancia, Argentina

${ }^{4}$ Universidad de la Costa, Colombia

${ }^{5}$ Universidad Maimónides, Argentina

${ }^{6}$ Intec, Instituto Tecnológico de Santo Domingo, República Dominicana

${ }^{7}$ Universidad de Concepción del Uruguay, Argentina

${ }^{8}$ Instituto de Neurociencia Cognitiva de Puerto Rico

${ }^{9}$ Universidad Corporativa Colombiana

\section{Resumen}

La situación de pandemia por COVID-19 motivó en todos los países la adopción de medidas extremas para evitar la propagación del virus. El cierre de escuelas, colegios y universidades cambió sorpresiva y radicalmente la actividad de docentes, estudiantes y familias; en pocas semanas los sistemas educativos debieron cambiar y adaptarse. La resiliencia docente fue la clave del éxito de esta transformación. Se estudió a 2.272 docentes de diversos países de lberoamérica a través de un cuestionario online diseñado para conocer los aspectos principales de su resiliencia, el impacto del COVID-19 en su condición de distanciamiento social, su soporte emocional, sus habilidades para la educación online, las emociones experimentadas y cambios en su estilo de vida, entre otros. Se utilizó la Escala de Resiliencia de Connor-Davinson (CD-RISC 10) como medida de resiliencia. A través de la RISC-10 se dividió la muestra en dos grupos, grupo de alta resiliencia (AR) y grupo de baja resiliencia (BR) y se obtuvieron medias y desvíos de cada ítem para cada grupo, además de la magnitud de efecto para observar el impacto de la diferencia en cada uno de ellos. Los resultados del estudio evidencian una asociación positiva entre los docentes con alta resiliencia y la percepción del evento disruptivo de la pandemia como una oportunidad, la percepción del soporte emocional brindado, la actividad física, la necesidad de formación en neuroeducación, el manejo de herramientas de educación online, las habilidades socioemocionales para enfrentar los cambios, la experiencia de emociones de valencia positiva y la instrumentación de cambios en el estilo de vida durante la pandemia. La resiliencia no solo es importante como recurso previo y de desarrollo personal del docente, sino también lo es como "acto pedagógico" al generar 
modelos de docentes capaces de enfrentar la adversidad desde una perspectiva de oportunidad de crecimiento.

\begin{abstract}
The pandemic situation due to COVID-19 motivated in all countries the adoption of extreme measures to prevent the spread of the virus. The closure of schools, colleges and universities surprisingly and radically changed the activities of teachers, students and families; in a few weeks the educational systems had to change and adapt. Teacher resilience was the key to the success of this transformation. 2272 teachers from different lberoamerican countries were studied through an online questionnaire designed to know the main aspects of their resilience, the impact of COVID-19 on their conditions of social distancing, their emotional support, their abilities for online education, the experienced emotions and changes in their lifestyle, among others. The Connor-Davinson Resilience Scale (CD-RISC 10) was used as a measure of resilience. Through the RISC-10, the sample was divided into two groups, the high-resilience group (AR) and the low-resilience group (BR), and means and deviations were obtained for each item for each group, in addition to the magnitude of effect for observe the impact of the difference in each of them. The results of the study show a positive association between teachers with high resilience and the perception of the disruptive event of the pandemic as an opportunity, the perception of the emotional support provided, physical activity, the need for training in neuroeducation, the management of online education, socio-emotional skills to cope with change, experiences of positive valence emotions, and the implementation of lifestyle changes during the pandemic. Resilience is not only important as a previous resource and for the teacher's personal development, but also as a "pedagogical act" by generating models of teachers capable of facing adversity from a growth opportunity perspective.
\end{abstract}

Palabras clave: resiliencia, docentes, COVID-19, neuroeducación, aislamiento, emociones, distanciamiento social, Iberoamérica

\section{Introducción}

\section{Resiliencia}

La resiliencia es objeto de estudio, con amplia literatura en los últimos años y de obligado conocimiento con la pandemia por COVID-19. La resiliencia es un constructo dinámico que alberga múltiples variables $^{1-4}$. Se entiende como un proceso de construcción social en el que median variables personales y ambientales $^{5}$. En el ámbito socioeducativo y psicosocial es un ejercicio diario que lleva a una nueva interpretación de los contextos frente a la adversidad a través de un cambio de mirada ${ }^{6,7}$ que abre la puerta a nuevas ideas e inspira transformaciones en las prácticas de intervención ${ }^{8}$, reforzando la eficacia y la eficiencia de los programas de ayuda y apoyo a la comunidad, trabajando en el refuerzo y desarrollo de potencialidades, más que en cubrir las carencias ${ }^{2,9-11}$.

En el ámbito educativo es clave a la hora de promover o activar procesos de resiliencia en los sujetos y comunidades educativas ${ }^{12}$. Para construir entornos más resilientes es fundamental invertir en el capital humano de estos entornos, ya sean los mismos niños y niñas como los maestros ${ }^{13,14}$ que permitan a quienes trabajan en ellos hacer posible lo imposible, generar respuestas resilientes y sostenibles en el tiempo 9 .

Existen dos conceptos clave sobre resiliencia que debemos diferenciar para el abordaje adecuado en el ámbito docente. Una es la "resiliencia clásica" que se refiere a la capacidad de un grupo o de una persona de afrontar, sobreponerse a las adversidades 
y resurgir fortalecido o transformado. La segunda, la "resiliencia generativa" se vincula con la virtud de generar opciones, metamorfosis y seguir viviendo?. La resiliencia generativa permite experimentar la adversidad como una oportunidad de crecimiento y desarrollo.

La esencia de este trabajo es identificar la situación de pandemia por COVID-19 como una oportunidad para investigar los aspectos que rodean a los docentes al enfrentar una situación inesperada, adversa, que obliga a la adaptación ${ }^{15}$. Pero también, busca contribuir en la identificación de la resiliencia como un acto pedagógico que demuestra a los estudiantes las actitudes, pensamientos, emociones y conductas que facilitan la resiliencia generativa para el desarrollo personal, social y comunitario ${ }^{16}$.

\section{La pandemia por COVID-19}

El 11 de marzo de 2020, la Organización Mundial de la Salud $(\mathrm{OMS})^{17}$, declaró el brote del nuevo coronavirus (SARS-CoV-2) como una pandemia, ya que la situación epidemiológica a escala internacional requirió la adopción de medidas inmediatas para hacer frente a esa emergencia. El mundo se encontró sorpresivamente frente a un "evento disruptivo de escala global" sin precedentes en la historia. La mayoría de los países tomaron como principal medida el "aislamiento social, preventivo y obligatorio" lo que implicó la restricción de desplazamientos y la recomendación de suspensión de actividades, a fin de disminuir la circulación y el contagio del Coronavirus (COVID-19); esto incluyó a las actividades en colegios y universidades transformando todos los sistemas educativos del mundo. La situación disruptiva generó un "tsunami" de diferentes estrategias para lograr una adaptación educativa rápida y adecuada al nuevo contexto de aislamiento social de la pandemia. Todos los protagonistas pusieron en juego su resiliencia, considerándola como concepto dinámico ${ }^{18}$, viéndose en la necesidad de implementar estrategias de afrontamiento para continuar con el año escolar, los estudiantes, las instituciones, las familias y, por supuesto, los docentes.

En los diferentes niveles y modalidades, maestros y profesores enfrentaron una situación inesperada y única. Para la gran mayoría, el aislamiento preventivo fue una experiencia nueva, lo cual significó un desafío que puso en juego las habilidades cognitivas, emocionales y sociales de todos. Si bien es un contexto adverso, también puede considerarse una oportunidad para conocernos, identificar y comprender los comportamientos propios y el de los demás.

Esta pandemia no solo cambió los sistemas educativos en todos los países, sino que impactó de lleno, de forma directa, intensa y sorpresiva en los docentes. Ante esta situación, nuestro grupo de investigación comenzó a diseñar varios estudios destinados a evaluar el impacto en tres grupos principales: estudiantes, familias y docentes. El presente estudio se centra en el último grupo, los docentes, acerca de quienes se formularon interrogantes sobre cómo percibían la situación, cuál había sido el impacto, cuán resilientes eran para enfrentar la situación, cuáles eran las estrategias implementadas, cuáles eran sus emociones y cómo percibían los aspectos relacionados con las condiciones de aislamiento.

\section{Aclaración}

La OMS ${ }^{19}$ recomendó el "aislamiento social" como medida de prevención, lo cual involucra disminuir al máximo la interacción con los otros. Esta disminución en el número y calidad de interacción social puede acarrear implícito un riesgo para las poblaciones más vulnerables. Si bien existen tres términos muy relacionados, es necesario diferenciarlos al describir situaciones o implementar medidas destinadas a la prevención.

En primer lugar, "aislamiento social" es "la disminución de la interacción social humana, manifestada por una baja en la cantidad y/o calidad de contactos, que genera sufrimiento a través de sentimientos y pensamientos percibidos como dolorosos y negativos". Definido así, el aislamiento social es una situación que se acompaña de la "soledad" como sentimiento doloroso, desadaptativo y disfuncional.

En segundo lugar, el concepto de "distanciamiento social" se refiere a la disminución de la interacción humana manifestada por la baja cantidad y/o calidad de contactos sin que se genere sufrimiento. Esta situación es aplicable a muchas situaciones en los cuales el individuo se distancia socialmente, pero ese distanciamiento no produce ni sufrimiento, ni consecuencias negativas en sus emociones, capacidad adaptativa y de funcionamiento.

Por último, el término "distanciamiento físico" se refiere a un aumento de la distancia corporal con 
el otro con la mínima afectación posible de la interacción social; por lo cual, el número de contactos y su calidad, si bien pueden modificarse, no alteran de manera significativa la salud mental como para producir consecuencias emocionales negativas $\mathrm{ni}$ desadaptación.

La diferencia entre el aislamiento social, el distanciamiento social y el distanciamiento físico se hizo más visible durante la pandemia de COVID-19, donde las redes sociales online y las comunicaciones nos acercaron más que en ningún momento de la historia, permitiendo aumentar la cantidad e incluso la calidad de la interacción. Este aspecto, si bien limitado, tiene dos contrapuntos a considerar, el primero, altamente positivo porque permitió continuar manteniendo nuestra estructura social de interacción. Y el segundo, requirió una adaptación extremadamente rápida y obligada, tomando por sorpresa a la población con sus diversos niveles de respuesta.

El 20 de marzo, a tan solo nueve días de la declaración de pandemia, la OMS llamó a conferencia de prensa para aclarar que el término "aislamiento" no era correcto y sugerir a los medios de comunicación y los gobiernos el uso de la palabra "distanciamiento" como medida de prevención.

\section{Objetivo del estudio}

El objetivo general de este estudio es evaluar la situación de los docentes, orientando la exploración de los pensamientos, emociones y personalidad que ayudan a implementar estrategias de resiliencia para enfrentar la pandemia de COVID-19. Los objetivos específicos del trabajo fueron: a) identificación de estrategias resilientes en docentes con distanciamiento social preventivo obligatorio de Iberoamérica durante la pandemia de COVID-19, y b) evaluación del impacto del distanciamiento social en docentes de Iberoamérica durante la pandemia de COVID-19.

\section{Materiales y métodos}

\section{Diseño:}

Estudio observacional, descriptivo, transversal y correlacional de identificación de estrategias resilientes y severidad de impacto en docente de Iberoamérica con distanciamiento social preventivo durante la pandemia de COVID-19 ${ }^{20}$.

\section{Población:}

La población se compuso por docentes de distintos países de Iberoamérica (Argentina, Colombia, Rep. Dominicana, Ecuador, España, Portugal, Panamá, Paraguay, Puerto Rico y Uruguay, entre otros), mayores de 18 años, que ejercen su actividad en cualquier nivel y modalidad educativa, convocados a través de redes sociales por instituciones $\mathrm{y} / \mathrm{o}$ referentes en educación de cada país pertenecientes a la Red Iberoamericana de Neurociencia Cognitiva (ver agradecimientos). Para participar era necesario tener acceso a una conexión de Internet o de datos y completar el cuestionario desde un computador o un teléfono móvil. Participaron un total de 2.272 docentes. Se aplicó la Escala de Resiliencia de Connor-Davinson (CD-RISC 10) ${ }^{21}$ usando la media de la escala M 32,49 como punto de corte. Se formaron dos grupos, aquellos con baja resiliencia (BR) $n=1201$ y los de alta resiliencia (AR) $n=1071$.

Instrumentos: Cuestionario "Resiliencia en docentes durante el distanciamiento social preventivo obligatorio por COVID-19"

Se solicitó a los participantes que completen el cuestionario de forma anónima. Las preguntas del cuestionario se orientaban a evaluar el impacto de la situación, los pensamientos, las emociones y los comportamientos de los docentes para enfrentar el período de distanciamiento social preventivo durante la pandemia de COVID-19.

La difusión del estudio comenzó el 8 de abril de 2020 y se mantuvo en línea durante veinte días. Cada participante podía completar el formulario solamente una vez.

El cuestionario se estructuró en cinco secciones, cada una de ellas contenía ítems de fácil respuesta a través de listas desplegables, opciones múltiples, casilla de verificación, escalas lineales o cuadrícula de opción múltiple. Constaba de 66 ítems con un tiempo estimado de respuesta de aproximadamente 10 minutos.

\section{Sección A: Consentimiento Informado}

Previo al comienzo del cuestionario los participantes fueron informados sobre el estudio a través de un consentimiento que cumple con los estándares éticos y resguardo de la información de los organismos y acuerdos internacionales. Cada docente debía leer y aceptar para continuar con su participación en el estudio. 


\section{Sección B: Datos Personales}

Esta sección consta de un total de 19 ítems divididos en dos partes. En la primera, se solicitaron datos sobre edad, género, país de residencia, estado civil, trabajo y nivel educativo y modalidad en donde desempeña su actividad docente. En la segunda, se preguntó sobre aspectos personales auto percibidos como formación, habilidades informáticas pre y post distanciamiento social preventivo, entre otras.

\section{Sección C: Resiliencia}

Esta sección está compuesta por 19 ítems divididos en dos clústeres de preguntas. El clúster A está conformado por preguntas referentes a la resiliencia ${ }^{22}$, a aspectos relacionados con la sorpresiva situación de pandemia por COVID-19. Las preguntas fueron formuladas por dos investigadores, una pedagoga y un psiquiatra, expertos en educación, resiliencia y salud mental. Para el clúster $B$ se seleccionó la Escala de Resiliencia de Connor-Davinson (CD-RISC 10) ${ }^{21}$ y dos preguntas seleccionadas de la Escala de felicidad subjetiva ${ }^{23,24}$.

Escala de Resiliencia CD-RISC 1021: es la versión breve de la CD-RISC en la adaptación española ${ }^{25}$. Está conformada por 10 ítems (los numerados como $1,4,6,7,8,11,14,16,17,19)$ de la escala original ${ }^{26}$. Mediante esta escala se solicita a los participantes que respondan en qué medida están de acuerdo con cada una de las frases que se les presenta (por ejemplo, el ítem 1: «Soy capaz de adaptarme a los cambios»). La forma de respuesta es una escala tipo Likert de cinco puntos desde 0 (totalmente en desacuerdo) hasta 4 (totalmente de acuerdo).

Escala Subjetiva de Felicidad ${ }^{23}$ : es una medida global de bienestar subjetivo que evalúa la felicidad como fenómeno psicológico global, considerando la definición de felicidad desde la perspectiva de quien responde. Si bien la escala consta de 4 ítems de frases sencillas, a los fines prácticos del nuestro estudio se seleccionaron los dos primeros por representar las dos medidas más significativas de felicidad subjetiva, la auto percibida y la felicidad comparada. El formato de respuesta en los ítems va desde una puntuación de 1 que indica Menos feliz hasta una puntuación de 7 que indica Más feliz.
Sección D: Condiciones del distanciamiento social preventivo

Consta de un total de 21 ítems orientados a las condiciones en las cuales el docente está viviendo el período de distanciamiento preventivo y obligatorio, por ejemplo, la cantidad de días de aislamiento, con quién lo realiza, con cuántos niños y adultos mayores, en cuántos metros cuadrados convive, si realiza ejercicio o no, si tiene algún factor de riesgo, si convive con personas con factores de riesgo, si consume sustancias o no, cuántas horas de exposición tiene a información sobre COVID-19, los cambios que implementó en su estilo de vida o la intensidad de las emociones vividas durante el distanciamiento.

\section{Sección E: Impacto del distanciamiento preventivo} Está conformada por 7 ítems orientados a recabar datos sobre el impacto vivido durante el período de distanciamiento social preventivo y obligatorio a través de aspectos como la ansiedad, percepción de contagio, percepción de la red de apoyo social primaria, y grado de tolerancia y optimismo frente a la situación.

\section{Análisis estadístico:}

Todos los datos se incorporaron en una base de datos. Una vez tabulados y practicado el control de calidad, los resultados se analizaron con el software $R$ v4.0.0 y RStudio v1.2.5033 y se utilizaron los paquetes readxl, table1, matchlt, ggplot2, ggpubr, effsize, tidyverse, corrplot, qwraps2 y magrittr. Los datos se presentan como medias, desvíos estándar, medianas y rango para los datos cualitativos y como frecuencias relativas y absolutas para los categóricos. La comparación de grupo se realizó mediante test t con corrección de Welch en casos de heterogeneidad de las varianzas, se recurrió al test $U$ de Mann Whitney cuando los datos se alejaron de la normalidad. El diagnóstico de normalidad se realizó mediante los test de Kolmogorov-Smirnov y Schapiro-Wilk. Los datos categóricos fueron contrastados mediante test de Chi cuadrado con corrección de continuidad de Fisher cuando fue necesario. Se realizó corrección por comparaciones múltiples por el método de Benjamini-Hochberg. Para todos los test se fijó un nivel de significancia en $p<0.01$. Se utilizó como medida de efecto la $D$ de Cohen para las variables continuas y el coeficiente $V$ de Cramer para las variables categóricas. Se reporta el estimado y el intervalo de confianza para el $99 \%(20,27)$. 


\section{Resultados}

\section{Características de la población docente}

El cuestionario fue completado por 2.272 personas en total, $91,7 \%$ mujeres y $8,3 \%$ varones, la edad tuvo una media de 41,1. En la Tabla 1 se puede observar las medias y desvíos estándar para el nivel en el que desarrollan su actividad docente, el tipo de institución en la que trabajan, las horas que dictan clase por semana.

Condiciones durante el distanciamiento social preventivo obligatorio

Se obtuvieron medias (M) y desvíos estándares (SD) para diferentes aspectos relacionados con las condiciones del distanciamiento social preventivo durante el período de COVID-19 y se reportan los valores de toda la muestra en forma global. Para el número de días en distanciamiento social se obtuvo un valor de M 30,9 (SD 8,43), para el ítem "número de personas con las que comparte el distanciamiento social" se obtuvo una M 3,03. El 19,2\% de los docentes presentaron uno o más factores de riesgo para COVID-19 y el $41,9 \%$ eran cuidadores de personas de riesgo. Con respecto a sus hábitos, el $29,5 \%$ no realiza ejercicio físico, el 33,5\% lo hace una a dos veces por semana, el $23,2 \%$ tres a cuatro por semana y el 13,8 cinco a siete veces semanales. El $71,5 \%$ no consume regularmente sustancias tóxicas, el 28,5\% restante consume en su mayoría alcohol $(19,1 \%)$ y tabaco $(11,7 \%)$, el consumo de marihuana, cocaína o drogas de diseño tuvo un reporte menor al $1 \%$; durante el distanciamiento social el $16,0 \%$ no varió el consumo, 7,3\% aumentó y el 6,0\% disminuyó el consumo de la sustancia referida. También durante la pandemia el $51 \%$ no tuvo cambios en su actividad sexual, el 29,3\% la disminuyó y el 16,5\% aumentó.

También se preguntó con respecto a las horas que le dedicaron a informarse en los medios de comunicación y redes sociales sobre la situación actualizada de la pandemia (Figura 1). Para todas estas variables no hubo diferencias significativas entre el grupo de BR y el grupo de AR.

Percepción del impacto, habilidades en educación online y formación.

Por otro lado, uno de los objetivos del estudio era observar cómo había impactado el distanciamiento social en la actividad docente. Acerca del sistema
Tabla 1. Características descriptivas de la población

\begin{tabular}{|c|c|}
\hline \multicolumn{2}{|c|}{$\begin{array}{c}\text { Total } \\
(\mathbf{N}=\mathbf{2 2 7 2})\end{array}$} \\
\hline \multicolumn{2}{|l|}{ Sexo } \\
\hline Masculino & $2084(91,7 \%)$ \\
\hline Femenino & $188(8,3 \%)$ \\
\hline \multicolumn{2}{|l|}{ Edad } \\
\hline Media (SD) & $41,1(8,91)$ \\
\hline Mediana (mín. - máx.) & $41,0(20,0-74,0)$ \\
\hline \multicolumn{2}{|l|}{ Estado civil } \\
\hline En pareja & $1450(63,8 \%)$ \\
\hline Divorciado/a & $201(8,8 \%)$ \\
\hline Soltero/a & $596(26,2 \%)$ \\
\hline Viudo/a & $25(1,1 \%)$ \\
\hline \multicolumn{2}{|l|}{ Nivel de enseñanza } \\
\hline Especial & $46(2,0 \%)$ \\
\hline Inicial & $360(15,8 \%)$ \\
\hline Primario & $726(32,0 \%)$ \\
\hline Secundario & $707(31,1 \%)$ \\
\hline Universitario & $392(17,3 \%)$ \\
\hline \multicolumn{2}{|l|}{ Trabaja en institución } \\
\hline Privada & $706(31,1 \%)$ \\
\hline Pública & $1525(67,1 \%)$ \\
\hline Desempleado & $24(1,1 \%)$ \\
\hline \multicolumn{2}{|c|}{ Horas de clase que dicta por semana } \\
\hline $0-10$ & $368(16,2 \%)$ \\
\hline $10-20$ & $598(26,3 \%)$ \\
\hline $20-30$ & $706(31,1 \%)$ \\
\hline 30- o más & $599(26,4 \%)$ \\
\hline
\end{tabular}

educativo, el $69,0 \%$ de los docentes respondieron que el COVID-19 había impactado positivamente, sin cambios el $16,0 \%$ y negativamente el $15,0 \%$. Además, se les preguntó cuánto mejorará la forma de enseñar y la forma de aprender de los estudiantes con la situación del COVID-19 (entre 1, nada y 10, totalmente) los docentes con puntajes más altos en

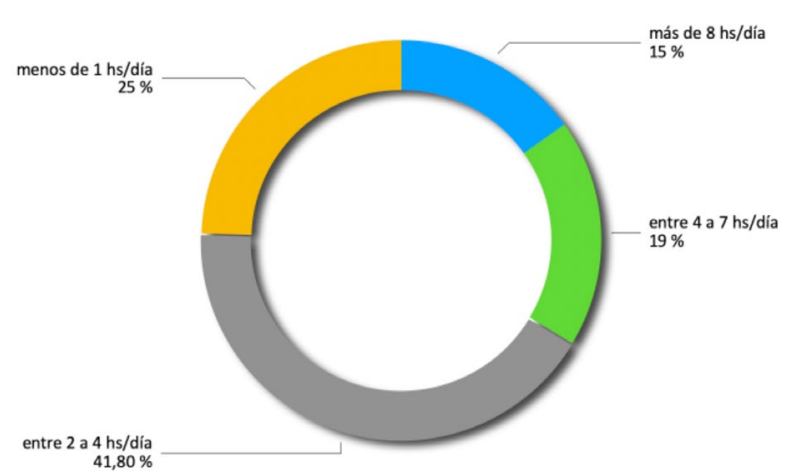

Figura 1. Cantidad de horas dedicadas a informarse en medios (TV, radio, periódicos) y redes sociales durante el distanciamiento social en COVID-19. 
la RISC-10 (Escala de resiliencia de Connor-Davinson) tuvieron una diferencia significativa $(p<0.001)$ a su favor frente a los docentes con bajos puntajes (Tabla 2).

Con respecto a las habilidades para el manejo de las herramientas de la educación online como, aplicaciones, plataformas educativas, mail, software de videoconferencias, etc., se investigó sobre la percepción de los docentes antes y actual del distanciamiento social; si bien todos los docentes aumentaron su percepción de habilidades al comparar los grupos con alta resiliencia (AR) en la RISC-10 y los de baja resiliencia (BR), se obtuvo una diferencia significativa entre ambos grupos. De igual manera ocurrió al preguntar sobre las habilidades de los estudiantes para el uso de herramientas de educación online. También, al consultar sobre cómo consideraban sus habilidades emocionales y sociales para enfrentar los cambios, nuevamente los docentes con alta AR presentaron diferencias significativas frente a los que tenían BR. Lo mismo ocurrió al consultar sobre las habilidades emocionales y sociales de los estudiantes.
Por último, se les solicitó el grado de formación en neuroeducación que tenían entre el grupo AR y el grupo BR. Y sobre si consideraban necesario una formación específica en neuroeducación sin diferencias significativas entre ambos grupos (Tabla 2 ).

\section{Percepción de soporte emocional}

Se investigó cuál era la percepción de los docentes con respecto al soporte que ellos brindaron a los estudiantes y a la comunidad durante el período de distanciamiento social, y cuál fue el brindado por la institución educativa en la que realizan su tarea docente habitualmente. Para un rango de 1, ningún apoyo, y 10, apoyo total, se obtuvieron medias y SD para el apoyo brindado por los docentes a los estudiantes, y para el apoyo brindado a la comunidad en general, los docentes AR obtuvieron una diferencia significativa en su puntaje en comparación al grupo de BR. Por otro lado, para el aspecto sobre la percepción del apoyo emocional recibido por la institución en la que trabajan ${ }^{28}$ se constató una diferencia significativa a favor del grupo con AR frente al grupo de BR (Tabla 3).

Tabla 2. Percepción de Impacto, habilidades en educación online y en neuroeducación en COVID-19

\begin{tabular}{|c|c|c|c|c|c|}
\hline & \multicolumn{5}{|c|}{ Índice de Resiliencia } \\
\hline & $\begin{array}{c}\text { Baja } \\
(\mathrm{N}=1201) \\
\text { Media (SD) }\end{array}$ & $\begin{array}{c}\text { Alta } \\
(\mathrm{N}=1071) \\
\text { Media (SD) }\end{array}$ & $\begin{array}{c}\text { Total } \\
(\mathrm{N}=2272) \\
\text { Media (SD) }\end{array}$ & p-valor & $\begin{array}{l}\text { M de } \\
\text { Efecto }\end{array}$ \\
\hline Mejorará la enseñanza & $7.32(1.87)$ & $7.94(1.76)$ & $7.61(1.84)$ & $<0.001$ & 0.03 \\
\hline Mejorará el aprendizaje & $6.92(2.01)$ & $7.52(1.94)$ & $7.21(2.00)$ & $<0.001$ & 0.30 \\
\hline Impacto en los próximos 5 años & $7.37(1.80)$ & $7.81(1.83)$ & $7.58(1.83)$ & $<0.001$ & 0.24 \\
\hline $\begin{array}{l}\text { Habilidades con herramientas de } \\
\text { educación online ANTES }\end{array}$ & $5.48(2.40)$ & $6.17(2.35)$ & $5.81(2.40)$ & $<0.001$ & 0.29 \\
\hline $\begin{array}{l}\text { Habilidades con herramientas de } \\
\text { educación online ACTUAL }\end{array}$ & $7.02(1.93)$ & $7.65(1.70)$ & $7.31(1.85)$ & $<0.001$ & 0.34 \\
\hline $\begin{array}{l}\text { Habilidades de los estudiantes con las } \\
\text { herramientas de educación online }\end{array}$ & $6.00(2.05)$ & $6.52(2.05)$ & $6.25(2.06)$ & $<0.001$ & 0.25 \\
\hline $\begin{array}{l}\text { Habilidades emocionales y sociales } \\
\text { para acompañar a los estudiantes }\end{array}$ & $7.30(1.56)$ & $8.12(1.30)$ & $7.68(1.50)$ & $<0.001$ & 0.57 \\
\hline $\begin{array}{l}\text { Habilidades emocionales y sociales } \\
\text { para afrontar los cambios }\end{array}$ & $7.38(1.54)$ & $8.15(1.33)$ & $7.75(1.50)$ & $<0.001$ & 0.53 \\
\hline Formación en neuroeducación & $4.24(2.57)$ & $4.86(2.74)$ & $4.53(2.67)$ & $<0.001$ & 0.23 \\
\hline $\begin{array}{l}\text { Necesidad de formación en } \\
\text { neuroeducación }\end{array}$ & $8.63(1.80)$ & $9.11(1.46)$ & $8.85(1.67)$ & $<0.001$ & 0.28 \\
\hline
\end{tabular}


Tabla 3. Percepción soporte emocional.

\begin{tabular}{|c|c|c|c|c|c|}
\hline & \multicolumn{5}{|c|}{ Índice de Resiliencia } \\
\hline & $\begin{array}{c}\text { Baja } \\
(\mathrm{N}=1201) \\
\text { Media (SD) }\end{array}$ & $\begin{array}{c}\text { Alta } \\
(\mathrm{N}=1071) \\
\text { Media (SD) }\end{array}$ & $\begin{array}{c}\text { Total } \\
(\mathrm{N}=2272) \\
\text { Media (SD) }\end{array}$ & p-valor & $\begin{array}{l}\text { M de } \\
\text { Efecto }\end{array}$ \\
\hline $\begin{array}{l}\text { Apoyo emocional brindado a los } \\
\text { estudiantes }\end{array}$ & $7.35(2.13)$ & $8.10(1.87)$ & $7.70(2.05)$ & $<0.001$ & 0.37 \\
\hline $\begin{array}{l}\text { Apoyo emocional brindado a la } \\
\text { comunidad }\end{array}$ & $7.09(2.24)$ & $7.89(2.00)$ & 7.47 (2.17) & $<0.001$ & 0,37 \\
\hline $\begin{array}{l}\text { Apoyo emocional recibido de la } \\
\text { institución }\end{array}$ & $5.68(2.73)$ & $6.36(2.78)$ & $6.00(2.78)$ & $<0.001$ & 0.24 \\
\hline
\end{tabular}

El distanciamiento social preventivo por COVID-19 como una oportunidad

En el estudio era clave saber si los docentes percibían esta situación disruptiva como una oportunidad para fortalecer su resiliencia y si habían profundizado la reflexión sobre distintos aspectos de su rol. Se exploró si durante el período de distanciamiento social habían reflexionado más sobre aspectos como su forma de enseñar y la forma de aprender de sus estudiantes, evidenciándose diferencias significativas entre los docentes con BR y los de AR en ambos ítems. Sin embargo, el punto central fue la percepción de los cambios como una oportunidad (Likert 0 a 4), obteniéndose una M 2.90 (SD 0,85) evidenciándose una diferencia significativa $p<0,001$ a favor el grupo AR frente al grupo BR. Sobre este ítem se aplicó $D$ Cohen para determinar la magnitud del efecto, 0.60 , siendo un excelente valor y el más alto obtenido durante el estudio. 0 sea, percibir los cambios como oportunidad fue el factor con más impacto para el desarrollo de la resiliencia docente (Tabla 4).

\section{Felicidad subjetiva}

Como mencionamos anteriormente, utilizamos los dos primeros ítems de la Escala de Felicidad Subjetiva ${ }^{23}$, el primer ítem sobre felicidad autoreportada y en el segundo ítem relacionado con la felicidad comparada con los otros. El grupo AR logró mejores resultados con una diferencia significativa a favor frente al grupo de BR en ambos ítems de la escala. Sobre estos puntos se aplicó D Cohen para medir el tamaño de efecto, para la felicidad auto reportada 0,52 , y 0,57 para la felicidad comparada, por lo cual se aprecia una magnitud del efecto "buena" para ambos ítems (Tabla 5).

Grado de intensidad emocional experimentada durante el distanciamiento social preventivo por COVID-19 Se investigó el grado de intensidad de emociones experimentadas por los docentes durante el distanciamiento social preventivo. Se estudiaron ocho emociones, cuatro emociones de valencia negativa, enojo, miedo, tristeza, escepticismo y cuatro emocio-

Tabla 4. Aislamiento preventivo por COVID-19 como oportunidad

\begin{tabular}{|c|c|c|c|c|c|}
\hline & \multicolumn{5}{|c|}{ Índice de resiliencia } \\
\hline & $\begin{array}{c}\text { Baja } \\
(\mathrm{N}=1201) \\
\text { Media (SD) }\end{array}$ & $\begin{array}{c}\text { Alta } \\
(\mathrm{N}=1071) \\
\text { Media (SD) }\end{array}$ & $\begin{array}{c}\text { Total } \\
(\mathrm{N}=2272) \\
\text { Media (SD) }\end{array}$ & p-valor & $\begin{array}{l}\text { M de } \\
\text { Efecto }\end{array}$ \\
\hline $\begin{array}{l}\text { Percepción de los cambios como } \\
\text { oportunidades }\end{array}$ & $2.67(0.799)$ & $3.17(0.839)$ & $2.90(0.855)$ & $<0.001$ & 0.60 \\
\hline Reflexión sobre la vida & $2.94(0.796)$ & $3.23(0.816)$ & $3.08(0.818)$ & $<0.001$ & 0.36 \\
\hline Reflexión sobre la enseñanza & $3.00(0.754)$ & $3.33(0.759)$ & $3.16(0.775)$ & $<0.001$ & 0.44 \\
\hline Reflexión sobre el aprendizaje & $3.04(0.729)$ & $3.41(0.658)$ & $3.21(0.720)$ & $<0.001$ & 0.52 \\
\hline
\end{tabular}


Tabla 5. Felicidad Subjetiva.

\begin{tabular}{lccccc}
\hline & \multicolumn{3}{c}{ Índice de resiliencia } \\
& $\begin{array}{c}\text { Baja } \\
(\mathrm{N}=1201) \\
\text { Media }(\mathrm{DS})\end{array}$ & $\begin{array}{c}\text { Alta } \\
(\mathrm{N}=1071) \\
\text { Media }(\mathrm{DS})\end{array}$ & $\begin{array}{c}\text { Total } \\
(\mathrm{N}=2272) \\
\text { Media }(\mathrm{DS})\end{array}$ & p-valor & $\begin{array}{c}\text { M de } \\
\text { Efecto }\end{array}$ \\
\hline Felicidad auto reportada & $7.75(1.57)$ & $8.54(1.46)$ & $8.12(1.57)$ & $<0.001$ & 0.52 \\
Felicidad comparada con los demás & $5.91(2.01)$ & $7.94(1.57)$ & $7.89(1.61)$ & $<0.001$ & 0.57 \\
\hline
\end{tabular}

nes de valencia positiva, compasión, empatía, amor y optimismo. El grupo de docentes AR obtuvo mejores resultados que los del grupo $B R$, con una diferencia significativa en casi todos los casos. Los tamaños de efectos calculados fueron en todos los casos bajos (Figura 2).

Cambios en el estilo de vida realizados durante distanciamiento social por COVID-19

Se indagó sobre qué cambios positivos en el estilo de vida que habían realizado durante el período de distanciamiento social preventivo podían señalar modificaciones en más de una actividad. El 100\% de los docentes reportaron al menos un cambio en su estilo de vida. El $27,8 \%$ realizó cambios positivos en su actividad física, el 30,4\% logró cambios positivos en su alimentación y el $34,4 \%$ en el sueño. En otras áreas, por ejemplo en la espiritualidad, lo hizo el $26,4 \%$ y el $21,7 \%$ implementó técnicas de relajación. El 38,4\% incrementó su actividad de estudio, el $37,7 \%$ incrementó la lectura y el $28,5 \%$ aumentó su interés por la música Los docentes tuvieron cambios en sus relaciones interpersonales, el $45,8 \%$ lograron

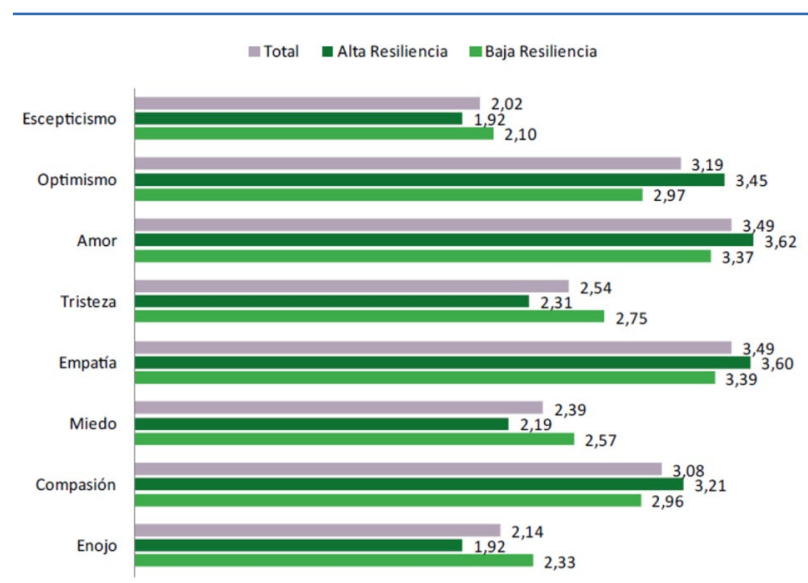

Figura 2. Medias del grado de emociones experimentadas durante el distanciamiento social en COVID-19. cambios positivos en las relaciones con sus hijos, el $31,6 \%$ lo hizo en las relaciones con su pareja y el $31,1 \%$ en otro tipo de relaciones interpersonales (padres, amigos, comunidad). Al comparar los grupos de BR y AR no se encontraron diferencias significativas (Figura 3).

\section{Discusión y conclusiones}

El objetivo del estudio fue medir el impacto de la situación de distanciamiento social preventivo y la resiliencia en docentes. Respondieron el cuestionario online 2.272 docentes de diversos países de Iberoamérica.

El $69 \%$ de los docentes consideró que la situación de COVID-19 tendrá un impacto positivo para el sistema educativo, lo cual puede ser interpretado de varias formas. En primer lugar, considerar un evento disruptivo como un factor de impacto positivo sobre la actividad docente podría estar relacionado con algunos aspectos de la resiliencia, esto coincide con otro resultado del cuestionario, como en el ítem "ver los cambios como oportunidad" donde se observa

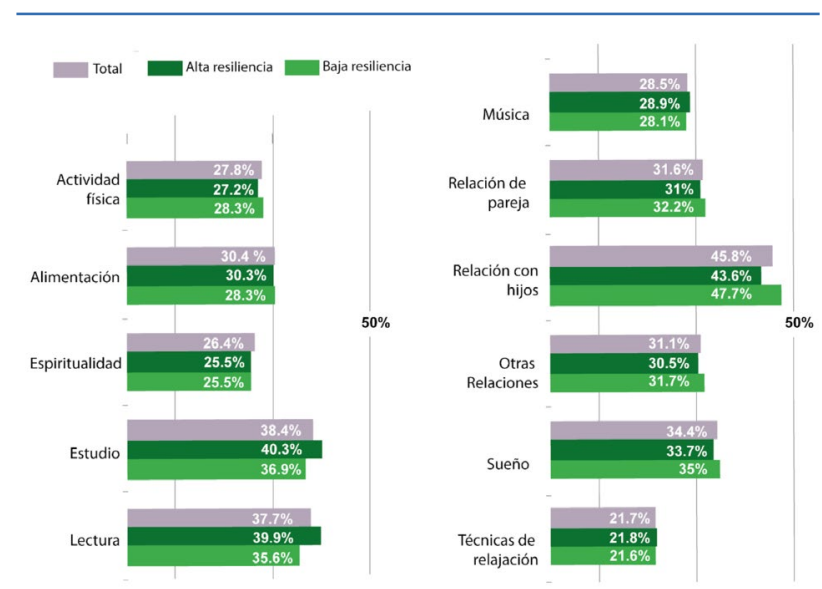

Figura 3. Cambios del estilo de vida docente durante el distanciamiento social. 
hubo una diferencia significativa a favor del grupo de AR comparado con el grupo de BR, al medir el tamaño de efecto de esta diferencia arrojó un valor 0,6 , además de ser un excelente tamaño de efecto, fue el de mayor tamaño evidenciado en el estudio. Es decir, el resultado apoya la consideración general de varios autores ${ }^{6-8}$ acerca de que considerar los cambios como oportunidad es uno de los factores más importante para la construcción de la resiliencia.

La situación de pandemia trajo aparejados cambios en la modalidad de enseñanza. La mayoría de las actividades, tanto en escuelas como en universidades, pasaron de la modalidad presencial a la modalidad online en pocas semanas. En el estudio se indagó sobre la percepción de los docentes sobre sus habilidades para el manejo de las herramientas de educación virtual como, por ejemplo, aplicaciones, plataformas educativas, mail, software de videoconferencias, etc. Se preguntó sobre la percepción "anterior" a la declaración del aislamiento preventivo, como la "actual", o sea en el momento de contestar el cuestionario. La percepción "anterior" M 5,81 $(S D 2,40)$ y "actual" M 7,31 (SD1,85) evidenciaron un aumento en todos los docentes. Sin embargo, el grupo con alta resiliencia (AR) obtuvo una diferencia significativa al compararlo con el grupo de baja resiliencia (RB), esta correlación puede implicar que la resiliencia podría ser un factor que facilite la adaptación. Otro dato obtenido del estudio es la percepción de los docentes respecto de su formación en "neuroeducación" M 4,53 (SD 2,67) una media relativamente baja, sin embargo, al preguntar sobre la necesidad de formación en neuroeducación se obtuvo una M 8,85 (SD 1,67). Es decir, si bien la formación actual es escasa, el reconocimiento de la necesidad de actualización en "neuroeducación" es muy alto al comparar ambos grupos en ambos ítems, los más resilientes reportan mayor necesidad de formación en neuroeducación $(p<0,001)$.

Con respecto a las condiciones del distanciamiento social preventivo, el único ítem que mostró resultados interesantes fue la actividad física. El 70,5\% de los docentes del estudio realizaron actividad física durante el distanciamiento social. La actividad física es reconocida como un factor asociado a mayor resiliencia en docentes ${ }^{29}$, al comparar los grupos hubo una diferencia significativa a favor del grupo de AR respecto del grupo de $B R$, si bien $V$ de Cramer fue baja.
Al investigar sobre la percepción del soporte emocional en dos aspectos, el brindado por el docente a sus estudiantes y a su comunidad, el grupo AR mostró mayor soporte emocional brindado a sus estudiantes y a su comunidad que los del grupo BR. Con los cual la resiliencia podría ser un factor que predispone y facilita a los docentes a brindar soporte emocional a los demás.

Cómo medida de bienestar se usaron dos ítems de la Escala Subjetiva de Felicidad ${ }^{23}$. El grupo de docentes con AR reportó puntajes más altos que los del grupo BR en el ítem "felicidad autoreportada". De igual manera sucedió en el ítem "felicidad comparada". Los puntajes altos en la RISC-10 (Escala de resiliencia) correlacionaron significativamente con los puntajes obtenidos en los ítems de felicidad autoreportada y felicidad comparada.

El estudio también investigó sobre la intensidad de las emociones experimentadas por los docentes durante el distanciamiento social. Las emociones de valencia negativa (escepticismo, enojo, miedo y tristeza) fueron experimentadas con menor intensidad que las emociones de valencia positiva, amor, empatía, optimismo y compasión, que lo hicieron con mayor intensidad. La tristeza fue la emoción con valencia negativa que alcanzó mayor intensidad reportada (M 2,54 SD 1,19). Dentro de las emociones con valencia positiva, la más intensamente experimentada fue el amor y la empatía, ambas con igual media $(M 3,49)$ y desvío estándar (SD 1,06). Los docentes con AR reportaron mayor intensidad en emociones de valencia positiva que los docentes con BR; inversamente, los docentes con $\mathrm{BR}$ reportaron mayor intensidad en emociones con valencia negativa. La alta resiliencia podría ser un factor que predispone a la experiencia de emociones de valencia positiva frente a la adversidad y la baja resiliencia lo hace hacia las emociones con valencia negativa.

Finalmente, la mayoría de los docentes implementaron cambios en su estilo de vida durante el distanciamiento social. Paradójicamente, la mayoría lo hizo acercándose a sus relaciones interpersonales más significativas como hijos, pareja, amigos y padres, no solo disminuyendo la distancia social, sino estrechando vínculos frente a la adversidad. Una vez más, señalamos que el "distanciamiento social" no es el término más adecuado para la recomendación de la $\mathrm{OMS}^{19}$, ya que, como queda demostrado, el vínculo social no solo se hace más cercano ante la adver- 
sidad sino que habitualmente es donde buscamos refugio. Las tecnologías de comunicación actuales nos permiten disminuir la distancia social y estrechar vínculos a pesar de las dificultades para encontrarnos. El término "distanciamiento físico" es el que recomendamos para una medida de prevención que solo busca evitar el contagio de un virus y no el aislamiento social del individuo o las familias.

Resumiendo, al revisar los resultados del estudio se evidencia una asociación positiva y significativa entre la "Alta Resiliencia" de los docentes y factores relevantes durante el distanciamiento social como la percepción del evento disruptivo de la pandemia como una oportunidad, la percepción del soporte emocional brindado, la actividad física, la necesidad de formación en neuroeducación, el manejo de herramientas de educación online, las habilidades socioemocionales para enfrentar los cambios, las experiencias de emociones de valencia positiva y la instrumentación de cambios en el estilo de vida durante la pandemia ${ }^{30}$.

La resiliencia debe desarrollarse en los docentes no solo como un factor protector de estrés crónico como está descrito ${ }^{31}$, sino como un factor que facilita la adaptación a los cambios, el soporte emocional, las relaciones interpersonales positivas como generador de bienestar incluso ante la adversidad ${ }^{32}$, estimulando el desarrollo personal y en comunidad. Sin olvidar, que en el contexto educativo "la resiliencia del docente" genera una acción pedagógica en los estudiantes que, a pesar de la adversidad, se presenta como una herramienta clave para generar climas emocionalmente positivos y más seguros para el aprendizaje $^{16}$.

\section{Limitaciones}

La limitación más importante del estudio surge de la modalidad de "cuestionario online", sin embargo, debido a la situación de pandemia y distanciamiento social, fue el recurso más apto para su realización. Esta modalidad puede acarrear un sesgo de voluntario, en donde se vean motivados a contestar quienes mejor estén pasando la situación. Si bien este sesgo puede afectar a los resultados globales, es esperable que se distribuya aleatoriamente entre los individuos con baja y alta resiliencia, por lo que su impacto sobre las diferencias entre grupos debería ser menor. La segunda limitación es el momento de la situación de distanciamiento social en que los participantes contestaron el cuestionario: las características fueron cambiando semana tras semana y el ánimo de la población es dependiente de estos cambios. La tercera limitación es la inclusión de varios países de lberoamérica, lo cual puede contener un sesgo en los resultados; sin embargo, al comparar los tres países que más participaron en el estudio, no se hallaron diferencias significativas en los resultados. Por último, el uso de una sola escala para evaluar resiliencia puede ser un factor limitante, si bien fue adecuada para la circunstancia y modalidad del presente estudio, se deberían incluir otros instrumentos en futuros trabajos.

\section{Agradecimientos}

Agradecemos a las dos instituciones impulsoras del estudio:

- Red Iberoamericana de Neurociencia Cognitiva

- Cátedra de Neuroeducación de la Universidad de Barcelona.

También agradecemos la colaboración en la difusión de las siguientes instituciones:

España:

- AIRE. Asociación para la investigación de la Resiliencia

Argentina:

- Fundación Hippocampus

- ABN. Aprendizaje Basado en Neurociencia

- Doctorado de la Universidad Maimónides en Psicología con orientación en Neurociencia Cognitiva Aplicada

- Universidad Católica de la Plata

- Universidad de Concepción del Uruguay

República Dominicana:

- Intec. Instituto Tecnológico de Santo Domingo

- Centro de Asistencia e Investigación Neurotraining

Colombia:

- Instituto Colombiano de Neuropedagogía

- Doctorado en Neurociencia Cognitiva Aplicada de la Universidad de la Costa.

- Universidad Cooperativa de Colombia, Campus Santa Marta, Facultad de Psicología.

Ecuador:

- Instituto de Neurociencia Feedback

Puerto Rico:

- Instituto de Neurociencia Cognitiva de Puerto Rico

Portugal:

- ICN Agency

- Psicosoma Editorial 


\section{Referencias}

1. Bronfenbrenner U. Ecology of the family as a context for human development: Research perspectives. Dev. Psych. 1986; 22(6); 723-742. doi: 10.1037/0012-1649.22.6.723

2. Forés A, Grané J. La resiliencia. Crecer desde la adversidad. Barcelona: Plataforma; 2008.

3. Luthar S, Brown P. Maximizing resilience through diverse levels of inquiry: prevailing paradigms, possibilities and priorities for the future. Dev. Psych. 2007; 19; 931-55.

4. Ungar M. The social ecology of resilience: addressing contextual and cultural ambiguity of a nascent construct. Am J Orthopsychiatry, 2011; 81; 1-17.

5. Madariaga JM (ed.). Nuevas miradas sobre la resiliencia: Ampliando ámbitos y prácticas. Barcelona: Gedisa; 2014.

6. Vanistendael S. Una definición sencilla de la resiliencia. París: BICE; 2014. Disponible en: http://bice.org/es/una-definicion-sencilla-de-la-resiliencia-por-stefanvanistendael/

7. Vanistendae S. La resiliencia o el realismo de la esperanza. París: BICE; 2016. Disponible en: https://bice.org/es/produit/ la-resiliencia-o-el-realismo-de-la-esperanza/

8. Hurtubia V. La resiliencia: de la inspiración a la acción. Guatemala: Oficina Internacional Católica de la Infancia; 2019.

9. Grané J, Forés A. Los patitos feos y los cisnes negros. Resiliencia y Neurociencia. Barcelona: Plataforma Editorial; 2019.

10. Ungar M. What Works: A Manual for Designing Programs that Build Resilience. Halifax: Resilience Research Centre; 2018.

11. Vargas G., Pérez S, Aldunce P. Aluviones y resiliencia en Atacama. Construyendo saberes sobre riesgos y desastres. Chile: Univ. De Chile; 2018. Disponible en: https://doi. org/10.34720/jr4r-6j31

12. Forés, A., Grané, J. La resiliencia en entornos socioeducativos. Madrid: Narcea; 2012.

13. Ungar M. Systemic Resilience: Principles and Processes for a Science of Change in Contexts of Adversity. Ec and Soc. 2018; 23(4); Art. 34

14. Day C, Gurr D. Leading Schools Successfully: Stories from the Field. Londres: Routledge; 2013.

15. Saavedra E. Investigación en resiliencia: Algunos estudios cualitativos y cuantitativos. Editorial Academia Española; 2011.

16. Serrano A, Sanz R. Reflexiones y propuestas prèacticas pra desarrollar la capacidad de resiliencia frente a los conflictos en la escuela. Publicaciones. 2019; 49(1); 177-190.

17. World Health Organization. Global Surveillance for human infection with coronavirus disease (COVID-19). InterimGuidance. Suiza: WHO; 2020. Disponible en: https://www.who. int/publicationsdetail/global-surveillance-for-human-infection-with-novel-coronavirus-(2019-ncov)

18. Rutter M. Resilience as a dynamic concept. Development and Psychopathology. 2012; 24 (2); 335-344.
19. World Health Organization. Infection prevention and control during health care when novel coronavirus ( $\mathrm{nCoV}$ ) infection is suspected. Interim Guidance. Genève, Suiza: WHO; 2020. Disponible en: https://www.who.int/publications-detail/infection-prevention-and-control-during-health-care-when-novelcoronavirus-(ncov)-infection-is-suspected-20200125

20. Hernández R, Fernández C, Baptista M. Metodología de la investigación ( $6^{\mathrm{a}}$ ed.). México DF: McGraw-Hill Interamericana; 2014.

21. Davidson JR. Connor-Davidson Resilience Scale (CD- RISC) Manual. Unpublished. 08-19-2018. Accessible at: www.cdrisc.com

22. Rubio JL, Puig G. Tutores de resiliencia: Dame un punto de apoyo y moveré MI mundo. Barcelona: Gesida; 2015.

23. Lyubomirsky S, Lepper H. A measure of subjective hapiness: preliminary reliability and construct validation. Social Ind. Res. 1999; 46; 137-155.

24. Vera-Villarroel P, Celis-Atenas K, Córdova-Rubio N. Evaluación de la felicidad: análisis psicométrico de la escala de felicidad subjetiva en población chilena. Ter Psico. 2011; vol. 29(1); 127-133.

25. Notario-Pacheco B, Solera M, Serrano MD, Bartolomé R, García-Campayo J, Martínez-Vizcaíno V. Reliability and validity of the Spanish version of the 10 item Connor-Davidson Resilience Scale (10 item CDRISC) in young adults. Health Quality Life Outcomes. 2011; 9, 63-68.

26. Connor KM, Davidson JR. Development of a new resilience scale: the Connor-Davidson Resilience Scale (CD-RISC). Depress Anxiety. 2003; 18(2); 76-82.

27. Miles MB, Huberman AM, Saldaña J. Qualitative data analysis: A methodssourcebook (3a ed.). Thousand Oaks, CA: Sage; 2014.

28. Olmo M, Segovia D. La resiliencia a partir de las relaciones profesionales en centro educativos desafiantes. Educação \& Formação. 2018; V3 (N9); 3-19.

29. Romero C. Meta-análisis del efecto de la actividad física en el desarrollo de la resiliencia. Retos. Nuevas Tendencias en Educación Física, Deporte y Recreación. 2015; (28); 98-103. Recuperado de: https://www.redalyc.org/articulo. oa?id=345741428019

30. Crespo M, Fernández-Lansac V, Soberón C. Spanish Version of the CD-RISC Resilience Scale for Chronic Stress Situations. Beh. Psych/Ps Cond. 2014; 22; 219-238.

31. Ungar M, Theron L. Resilienceand mental health: how multisystemic processes contribute to positive outcomes. The lancet. Psychiatry. 2020; 7(5); 441-448.

32. Vicente de Vera MI, Gabari MI. La resiliencia como factor protector del estrés crónico en docentes. EuropJourlnvestHealth, Psychol and Educ. 2019; Vol. 9(3); 159-175. 